\title{
ROBUST CONTROL OF DISCRETE-TIME HYBRID SYSTEMS WITH UNCERTAIN MODAL DYNAMICS
}

\author{
THORDUR RUNOLFSSON
}

Received 26 April 2004

We study systems that are subject to sudden structural changes due to either changes in the operational mode of the system or failure. We consider linear dynamical systems that depend on a modal variable which is either modeled as a finite-state Markov chain or generated by an automaton that is subject to an external disturbance. In the Markov chain case, the objective of the control is to minimize a risk-sensitive cost functional. The risksensitive cost functional measures the risk sensitivity of the system to transitions caused by the random modal variable. In the case when a disturbed automaton describes the modal variable, the objective of the control is to make the system as robust to changes in the external disturbance as possible. Optimality conditions for both problems are derived and it is shown that the disturbance rejection problem is closely related to a certain risksensitive control problem for the hybrid system.

\section{Introduction}

In this paper, we study systems that are subject to sudden structural changes due to either changes in the operational mode of the system or failure. In particular, we study linear dynamical systems that operate in several modes. The modal variable determines the operational mode of the system and may be generated by some kind of a supervisory system, it may be random, or it may be some combination of the two. Systems of this form arise in various applications and system formulations, such as power systems [20], target tracking [11], and fault-tolerant control systems $[11,16]$.

Control of hybrid systems, where the modal variable is modeled as a random process, has been studied by many authors. In [17], a theory for linear hybrid systems with a Markovian jump parameter (modal variable) is developed and it is shown that an optimal state feedback control law in the case of a quadratic cost functional is given by a system of coupled Riccati equations. In [11], the theory for jump linear systems with a quadratic cost functional is developed further and the theory for such systems is quite complete. In $[7,8]$, a detailed treatment of continuous-time nonlinear stochastic hybrid systems is given. In particular, in [7], conditions for the existence and uniqueness of solutions of 
such systems are formulated, and in [8], a general theory for the ergodic properties of solutions is developed.

Hybrid systems, where the modal variable is assumed to evolve according to the dynamics of a finite-state machine (automaton), have recently been the subject of considerable research efforts. In particular, the stability properties of such systems have been studied in many publications (see, e.g., $[2,12,21]$ ). The design of stabilizing controllers for hybrid systems has been discussed in several publications (see, e.g., [19]) and optimal control for a class of hybrid systems has been studied in [13]. In addition, the design of supervisory control systems for hybrid systems has been the subject of several papers (see, e.g., [3]).

Risk-sensitive control has been the subject of many publications in the last 15-20 years. Initially, efforts focused on risk-sensitive control of linear systems in both continuous and discrete time (see [18] for an overview). After the paper [9] linking robust and risk-sensitive control for linear discrete-time systems, a considerable effort was made in analyzing this relationship further and a complete solution for the connection between risk-sensitive control, stochastic differential games, and robust control was obtained for diffusions in $[6,15]$. Risk-sensitive control has since then been developed for other types of systems. In particular, risk-sensitive control for Markov chain systems has been analyzed in several publications (see, e.g., $[1,5,10,14]$ ). Of special importance for our research are $[1,5]$, where robust control of finite-state machines is linked to risk-sensitive control of Markov chains on a finite-state space. In particular, our approach is partially based on ideas in these publications.

In this paper, we consider both systems where the modal variable is modeled as a finitestate Markov chain and systems where the modal variable is generated by an automaton that is subject to an external disturbance. In the Markov chain case, the objective of the control is to minimize a risk-sensitive cost functional. The risk-sensitive cost functional measures the risk sensitivity of the system to transitions caused by the random jump parameter (mode variable). For the case when the modal variable is described by a disturbed automaton, the objective of the control is to make the system as robust to changes in the external disturbance as possible. Optimality conditions for both problems are derived and it is shown that the disturbance rejection problem is linked (equivalent in the appropriate sense) to a certain risk-sensitive control problem for a hybrid system. The paper is organized as follows. In Section 2, the hybrid system that we study is described. In Section 2.1, the risk-sensitive control problem is discussed, and in Section 2.2, we analyze the disturbance rejection problem. Finally, in Section 3, we discuss the relationship between the two problems.

\section{System formulation}

Consider the linear system

$$
x_{k+1}=A\left(r_{k}\right) x_{k}+B\left(r_{k}\right) u_{k}, \quad x_{0}=x,
$$

where $x_{k} \in \mathbb{R}^{n}$ is the state and $u_{k} \in \mathbb{R}^{m}$ is the control. The variable $r_{k}$, the mode of the system, takes values in the finite set $M=\left\{m^{1}, \ldots, m^{N}\right\}$ and may evolve either deterministically or stochastically. The dynamics of $r_{k}$ are further specified in Sections 2.1 and 2.2. 
We define the class $\mathfrak{U}$ of admissible controls for system (2.1) as the class of all state feedback controls of the form $u=u(x, r)$, which satisfy a Lipschitz condition in $x$ uniformly in $r$ and for which $u(0, r)=0$ for all $r \in M$.

2.1. Risk-sensitive problem. We now assume that the mode variable $r_{k}$ in (2.1) is modeled as a Markov chain taking values in the finite set $M=\left\{m^{1}, \ldots, m^{N}\right\}$. In particular, assume that $r_{k}$ evolves according to the dynamics

$$
P\left(r_{k+1}=\hat{m} \mid r_{k}=m\right)=\Pi(m, \hat{m})
$$

where the stochastic matrix $\Pi=(\Pi(m, \hat{m}))_{m, \hat{m}=1}^{M}$ satisfies $\Pi(m, \hat{m}) \geq 0$ and $\sum_{\hat{m} \in M} \Pi(m$, $\hat{m})=1$.

For the stochastic system with state $\left(x_{k}, r_{k}\right)$, define the infinite-horizon risk-sensitive cost functional

$$
J(u)=\limsup _{K \rightarrow \infty} \frac{1}{K} \log E_{x, r}\left[e^{\sum_{k=0}^{K-1} c\left(x_{k}, r_{k}, u_{k}\right)}\right],
$$

where $c(x, r, u)=(1 / 2)\left(x^{\prime} Q(r) x+u^{\prime} R(r) u\right), Q(r) \geq 0, R(r)>0$, and the initial state is $\left(x_{0}, r_{0}\right)=(x, r)$. The objective of the control is to minimize the cost functional $J(u)$ over the set of admissible controls.

The stochastic system with state vector $\left(x_{k}, r_{k}\right)$ and control $u \in \mathfrak{U}$ is a Markov process. Note that if $X \subseteq \mathbb{R}^{n}$ and $S \subseteq M$, then

$$
P\left(\left(x_{k+1}, r_{k+1}\right) \in X \times S \mid\left(x_{k}, r_{k}\right)=(x, r)\right)=\sum_{\hat{r} \in S} \Pi(r, \hat{r}) \chi_{X}(A(r)+B(r) u),
$$

where $\chi_{X}(x)$ is the indicator function for the set $X$. For $\phi: \mathbb{R}^{n} \times M \rightarrow \mathbb{R}$, define

$$
\begin{gathered}
(P \phi)(x, r)=\sum_{\hat{r} \in M} \Pi(r, \hat{r}) \phi(A(r)+B(r) u, \hat{r}), \\
\left(P^{c} \phi\right)(x, r)=e^{c(x, r, u)}(P \phi)(x, r)=e^{c(x, r, u)} \sum_{\hat{r} \in M} \Pi(r, \hat{r}) \phi(A(r)+B(r) u, \hat{r}) .
\end{gathered}
$$

Theorem 2.1. Assume that there exist a constant $\lambda$ and a strictly positive function $\psi: \mathbb{R}^{n} \times$ $M \rightarrow \mathbb{R}$ such that

$$
e^{\lambda} \psi(x, r)=\inf _{u}\left(P^{c} \psi\right)(x, r), \quad(x, r) \in \mathbb{R}^{n} \times M .
$$

Then, for any $u \in \mathfrak{U}$,

$$
\lambda \leq J(u)=\limsup _{K \rightarrow \infty} \frac{1}{K} \log E_{x, r}\left[e^{\sum_{k=0}^{K-1} c\left(x_{k}, r_{k}, u_{k}\right)}\right] .
$$

Furthermore, if there exists a $u^{*} \in \mathfrak{U}$ such that the infimum in (2.6) is attained at $u^{*}$ for all $(x, r) \in \mathbb{R}^{n} \times M$, then

$$
\lambda=J\left(u^{*}\right)=\limsup _{K \rightarrow \infty} \frac{1}{K} \log E_{x, r}\left[e^{\sum_{k=0}^{K-1} c\left(x_{k}, r_{k}, u_{k}^{*}\right)}\right] .
$$


Proof. Note that for any $u \in \mathfrak{U}$ and $(x, r) \in \mathbb{R}^{n} \times M$,

$$
e^{\lambda} \psi(x, r) \leq\left(P^{c} \psi\right)(x, r)=e^{c(x, r, u)} \sum_{\hat{r} \in M} \Pi(r, \hat{r}) \psi(A(r)+B(r) u, \hat{r}) .
$$

For $R>0$, define

$$
\psi_{R}(x, r)= \begin{cases}\psi(x, r) & \text { if }|x| \leq R \\ 0 & \text { otherwise }\end{cases}
$$

Then,

$$
e^{\lambda} \psi_{R}(x, r) \leq\left(P^{c} \psi_{R}\right)(x, r)
$$

Therefore,

$$
e^{c(x, r, u)} \geq \frac{e^{\lambda} \psi_{R}(x, r)}{\sum_{\hat{r} \in M} \Pi(r, \hat{r}) \psi_{R}(A(r)+B(r) u, \hat{r})}=\frac{e^{\lambda} \psi_{R}(x, r)}{\left(P \psi_{R}\right)(x, r)},
$$

and thus

$$
\begin{aligned}
E_{x, r}\left[e^{\sum_{k=0}^{K-1} c\left(x_{k}, r_{k}, u_{k}\right)}\right] & \geq E_{x, r}\left[\prod_{k=0}^{K-1} \frac{e^{\lambda} \psi_{R}\left(x_{k}, r_{k}\right)}{\left(P \psi_{R}\right)\left(x_{k}, r_{k}\right)}\right] \\
& =\left(e^{\lambda}\right)^{K} E_{x, r}\left[\prod_{k=0}^{K-1} \frac{\psi_{R}\left(x_{k}, r_{k}\right)}{\left(P \psi_{R}\right)\left(x_{k}, r_{k}\right)}\right] .
\end{aligned}
$$

Taking logarithms of both sides of (2.13) and dividing through by $K$ gives

$$
\frac{1}{K} \log E_{x, r}\left[e^{\sum_{k=0}^{K-1} c\left(x_{k}, r_{k}, u_{k}\right)}\right] \geq \lambda+\frac{1}{K} E_{x, r}\left[\prod_{k=0}^{K-1} \frac{\psi_{R}\left(x_{k}, r_{k}\right)}{\left(P \psi_{R}\right)\left(x_{k}, r_{k}\right)}\right] .
$$

Noting that $\left(P \psi_{R}\right)\left(x_{k}, r_{k}\right)=E_{x_{k}, r_{k}}\left[\psi_{R}\left(x_{k+1}, r_{k+1}\right)\right]$, it follows from the Markov property of $\left(x_{k}, r_{k}\right)$ and standard properties of conditional expectations that

$$
E_{x, r}\left[\prod_{k=0}^{K-1} \frac{\psi_{R}\left(x_{k}, r_{k}\right)}{\left(P \psi_{R}\right)\left(x_{k}, r_{k}\right)}\left(P \psi_{R}\right)\left(x_{K-1}, r_{K-1}\right)\right]=\psi_{R}(x, r) .
$$

Then, since $\psi_{R}$ is bounded and strictly positive, there exist positive constants $C_{1}$ and $C_{2}$ (dependent on $R$ ) such that

$$
C_{1} \leq \frac{\psi_{R}(x, r)}{\sup _{x, r}\left(P \psi_{R}\right)(x, r)} \leq E_{x, r}\left[\prod_{k=0}^{K-1} \frac{\psi_{R}\left(x_{k}, r_{k}\right)}{\left(P \psi_{R}\right)\left(x_{k}, r_{k}\right)}\right] \leq \frac{\psi_{R}(x, r)}{\inf _{x, r}\left(P \psi_{R}\right)(x, r)} \leq C_{2} .
$$

It follows from (2.16) that the last term in (2.14) converges to zero as $K \rightarrow \infty$ and the result follows. Furthermore, if the infimum in (2.6) is attained at $u^{*}$ for all $(x, r) \in \mathbb{R}^{n} \times M$, then (2.8) follows by replacing the inequality in (2.9) (evaluated at $u^{*}$ ) by equality and repeating the above arguments. 
Consider (2.6) again, that is,

$$
e^{\lambda} \psi(x, r)=\inf _{u}\left(P^{c} \psi\right)(x, r)=\inf _{u} e^{c(x, r, u)}(P \psi)(x, r) .
$$

Let $\phi=\log \psi$. Then, after substituting into (2.17) and taking logarithms of both sides, we get (note that since the log is monotone increasing, we can interchange the inf and the $\log )$

$$
\begin{aligned}
\lambda+\phi(x, r) & =\inf _{u \in \mathfrak{U}}\left\{c(x, r, u)+\log \left(P e^{\phi}\right)(x, r)\right\} \\
& =\inf _{u \in \mathfrak{U}}\left\{c(x, r, u)+\log \sum_{\hat{r} \in M} \Pi(r, \hat{r}) e^{\phi(A(r) x+B(r) u, \hat{r})}\right\} .
\end{aligned}
$$

Next, we rewrite (2.18) as an equivalent stochastic game for an auxiliary system. This formulation will be the key in obtaining the link between the disturbance rejection and risk-sensitive control problems. We begin by introducing some notation. Let $\mathscr{P}(M)$ denote the set of all probability vectors on $M$, that is,

$$
\mathscr{P}(M)=\left\{p=(p(1), \ldots, p(N)): p(i) \geq 0, \sum_{i=1}^{N} p(i)=1\right\} .
$$

For a fixed $p \in \mathscr{P}(M)$, define the relative entropy function $I(\cdot \mid p): \mathscr{P}(M) \rightarrow \mathbb{R} \cup\{\infty\}$ by

$$
I(\pi \| p)= \begin{cases}\sum_{\hat{r} \in M} \log [\alpha(\hat{r})] \pi(\hat{r}) & \text { if } \pi \ll p, \\ \infty & \text { otherwise, }\end{cases}
$$

where

$$
\alpha(\hat{r})= \begin{cases}\frac{\pi(\hat{r})}{p(\hat{r})} & \text { if } p(\hat{r})>0 \\ 1 & \text { otherwise. }\end{cases}
$$

It is straightforward to prove the following lemma using the results in [4].

Lemma 2.2. Assume that $\phi(x, r)$ is bounded as a function of $r$ for each fixed $x$. Then

$$
\begin{aligned}
\log \sum_{\hat{r} \in M} \Pi(r, \hat{r}) e^{\phi(A(r) x+B(r) u, \hat{r})} \\
\quad=\sup _{\pi \in \mathscr{P}(M)}\left\{\sum_{\hat{r} \in M} \phi(A(r) x+B(r) u, \hat{r}) \pi(\hat{r})-I(\pi \| \Pi(r, \cdot))\right\} .
\end{aligned}
$$

Furthermore, the maximum of the right-hand side is attained at the unique probability measure

$$
\pi_{x}^{*}(r, \hat{r})=\frac{e^{\phi(A(r) x+B(r) u, \hat{r})}}{\left(P e^{\phi}\right)(x, r)} \Pi(r, \hat{r})
$$


Let $f^{u}(x, r)=A(r) x+B(r) u$. Substituting (2.22) into (2.18) gives

$$
\begin{aligned}
\lambda+\phi(x, r) & =\inf _{u}\left\{c(x, r, u)+\sup _{\pi \in \mathscr{P}(M)}\left\{\sum_{\hat{r} \in M} \phi\left(f^{u}(x, r), \hat{r}\right) \pi(\hat{r})-I(\pi \| \Pi(r, \cdot))\right\}\right\} \\
& =\inf _{u} \sup _{\pi \in \mathscr{P}(M)}\left\{\sum_{\hat{r} \in M} \phi\left(f^{u}(x, r), \hat{r}\right) \pi(\hat{r})+c(x, r, u)-I(\pi \| \Pi(r, \cdot))\right\} .
\end{aligned}
$$

This equation is the optimality equation for an associated stochastic dynamic game with dynamics given by

$$
x_{k+1}=A\left(s_{k}\right) x_{k}+B\left(s_{k}\right) u_{k},
$$

where $s_{k}$ is a process on $M$ which moves to the next state $s_{k+1}$ according to the probability distribution $\pi$. Furthermore, the probability distribution $\pi$ is the maximizing player in the differential game, the minimizing player is the control $u$, the one-stage reward is $c(x, r, u)-I(\pi \| \Pi(r, \cdot))$, and the cost functional is the infinite-horizon average cost

$$
J(u, \pi)=\limsup _{K \rightarrow \infty} E_{x, r}\left[\frac{1}{K} \sum_{k=0}^{K-1}\left(c\left(x_{k}, r_{k}, u_{k}\right)-I\left(\pi_{k} \| \Pi\left(r_{k}, \cdot\right)\right)\right)\right] .
$$

2.2. Disturbance rejection problem. We consider again the linear system

$$
x_{k+1}=A\left(m_{k}\right) x_{k}+B\left(m_{k}\right) u_{k}, \quad x_{0}=x,
$$

where, as before, $x \in \mathbb{R}^{n}$ is the state and $u \in \mathbb{R}^{m}$ is the control. The variable $m_{k}$, the mode of the system, is now modeled as a finite-state machine or automaton taking values in the finite set $M=\left\{m^{1}, \ldots, m^{N}\right\}$ and it evolves according to the dynamics

$$
m_{k+1}=g\left(m_{k}, w_{k}\right),
$$

where $w_{k}$ is a disturbance that belongs to a finite set $W$. We assume that there exists a null disturbance $w^{0} \in W$ such that $g\left(m, w^{0}\right)=h(m)$. The dynamics

$$
m_{k+1}=h\left(m_{k}\right)
$$

are called the undisturbed dynamics. We assume that for (2.29), there exists an equilibrium state $\bar{m} \in M$ such that $\bar{m}=h(\bar{m})$.

We use the notation $[0, K]$ for the time interval $\{0,1, \ldots, K\}$ and if $X$ is a set, the notation $X[0, K]$ is used for the set of functions $\bar{x}:[0, K] \rightarrow X$. We make the following assumptions about systems (2.1) and (2.2).

(A) There exists a $K_{0}$ such that for any $m_{0}$, the undisturbed system reaches the equilibrium state $\bar{m}$ after $K_{0}$ steps, that is, $\bar{m}=m_{K_{0}}=h\left(m_{K_{0}-1}\right)$ for any initial state $m_{0}$.

(B) Given $m, \hat{m} \in M$, there exist a $K_{1}$ and $\bar{w} \in W\left[0, K_{1}\right]$ such that for $m_{0}=m$ and input $\bar{w}$, the disturbed system reaches $\hat{m}$ in $K_{1}$ steps, that is, $\hat{m}=m_{K_{1}}=g\left(m_{K_{1}-1}\right.$, $w_{K_{1}-1}$ ). 
Remark 2.3. Assumption (A) is clearly a stability condition for the equilibrium state $\bar{m}$ of the undisturbed finite-state machine. Assumption (B) is a reachability condition for the disturbed finite-state machine, that is, any state in $M$ can be reached in finite time from any initial state by an appropriate choice of the disturbance input.

We make the following stability definitions for the hybrid system (2.27).

Definition 2.4. The equilibrium point $x=0$ of the hybrid system (2.27) with control $u_{k}=$ $u\left(x_{k}, m_{k}\right) \in \mathfrak{U}$ is said to be

(i) uniformly stable if there exists a finite constant $\gamma>0$ such that for any $k_{0}$ and $x_{0}$, the solution of (2.27) satisfies

$$
\left\|x_{k}\right\|<\gamma\left\|x_{0}\right\|, \quad k \geq k_{0}
$$

(ii) uniformly asymptotically stable if it is uniformly stable and $\left\|x_{k}\right\| \rightarrow 0$ as $k \rightarrow \infty$.

Remark 2.5. Stability of hybrid systems of the form (2.27), (2.28), and (2.29) has recently been extensively studied in the literature. Most of the approaches involve constructing families of Lyapunov functions that guarantee stability. An extensive overview of these methods can be found in [12].

Consider the closed-loop system with control $u_{k}=u\left(x_{k}, m_{k}\right) \in \mathfrak{U}$ and define

$$
f^{u}\left(x_{k}, u_{k}\right)=A\left(m_{k}\right) x_{k}+B\left(m_{k}\right) u\left(x_{k}, m_{k}\right)
$$

We make the following stability assumption.

(C) The system with dynamics $x_{k+1}=f^{u}\left(x_{k}, \bar{m}\right)$ is asymptotically stable.

Remark 2.6. Note that, in particular, we assume that the undisturbed system with initial state $(x, \bar{m})$ is asymptotically stable.

Lemma 2.7. Assume (A) and (C). Then the undisturbed hybrid system (2.27), (2.29) is uniformly asymptotically stable.

Proof. Let $K_{0}$ be as defined in assumption (A). Then

$$
x_{k+1}= \begin{cases}f\left(x_{k}, m_{k}\right) & k \in\left[0, K_{0}-1\right], \\ f\left(x_{k}, \bar{m}\right) & k \geq K_{0} .\end{cases}
$$

Note that

$$
\begin{aligned}
\left\|f^{u}(x, m)\right\| & =\|A(m) x+B(m) u(x, m)\| \leq\left(\|A(m)\|+\|B(m)\| l^{u}\right)\|x\| \\
& =C(m)\|x\|,
\end{aligned}
$$

where $l^{u}$ is the Lipschitz constant for $u(x, m)$. Define $\bar{C}=\max _{m \in M} C(m)<\infty$ and let $\hat{\gamma}=$ $\max \left(1, \bar{C}, \bar{C}^{K_{0}-1}\right)$. Then it is straightforward to see that

$$
\left\|x_{k}\right\| \leq \hat{\gamma}\left\|x_{0}\right\|, \quad k \in\left[0, K_{0}-1\right] .
$$


By (2.32) and assumption (C), we know that for $k \geq K_{0}$, there exists a $\tilde{\gamma}>0$ so that

$$
\left\|x_{k}\right\| \leq \tilde{\gamma}\left\|x_{0}\right\|, \quad k \geq K_{0}
$$

and $\left\|x_{k}\right\| \rightarrow 0$ as $k \rightarrow \infty$.

We associate with the disturbance $w_{k}$ in (2.28) a cost function $d(w)$, which satisfies

$$
\begin{gathered}
d\left(w^{0}\right)=0, \\
d(w)>0, \quad w \neq w^{0} .
\end{gathered}
$$

Define the quadratic running cost function $c(x, m, u)$ as in Section 2.1. The robust control problem or disturbance rejection problem we consider is the following.

Let $x_{0}=0, m_{0}=\bar{m}$. For a given constant $\gamma$, find a control $u$ (if it exists) such that

$$
\sum_{k=0}^{K-1} c\left(x_{k}, m_{k}, u_{k}\right) \leq \gamma^{2} \sum_{k=0}^{K-1} d\left(w_{k}\right)
$$

for all $\bar{w} \in W[0, K-1], K>0$.

For a given $u$, define $\gamma^{*}(u)$ as the smallest positive number $\gamma$ such that for all $\bar{w} \in$ $W[0, K-1], K \geq 1$, and $x_{0}=0, m_{0}=\bar{m},(2.37)$ holds. It can be shown that $\gamma \geq \gamma^{*}(u)$ if and only if there exists a nonnegative function $\Phi(x, m)$ (a so-called storage function; see [5]) such that

$$
\begin{gathered}
\Phi(x, m) \geq \max _{w \in W}\left[\Phi\left(f^{u}(x, m), g(m, w)\right)+c(x, m, u)-\gamma^{2} d(w)\right], \\
\Phi(0, \bar{m})=0 .
\end{gathered}
$$

In system (2.27), (2.28), let the control $u \in \mathfrak{U}$ be fixed and consider the system

$$
\begin{aligned}
& x_{k+1}=f^{u}\left(x_{k}, m_{k}\right), \quad x_{0}=x, \\
& m_{k+1}=g\left(m_{k}, w_{k}\right), \quad m_{0}=m \text {. }
\end{aligned}
$$

For this system, define a cost functional

$$
L(\bar{w})=\limsup _{K \rightarrow \infty} \frac{1}{K} \sum_{k=0}^{K-1}\left(c^{u}\left(x_{k}, m_{k}\right)-\gamma^{2} d\left(w_{k}\right)\right),
$$

where $c^{u}(x, m)=c(x, m, u)$, and consider the optimal control problem of maximizing $L(\bar{w})$ over all admissible disturbances $\bar{w} \in W[0, \infty)$. The dynamic programming equation for this problem is

$$
\lambda+\phi(x, m)=\max _{w \in W}\left[\phi\left(f^{u}(x, m), g(m, w)\right)+c^{u}(x, m)-\gamma^{2} d(w)\right] .
$$

Using standard dynamic programming arguments, it is straightforward to obtain the following. 
Theorem 2.8. Assume that there exist a $\lambda \geq 0$ and a function $\phi$ so that (2.41) is satisfied for all $(x, m) \in \mathbb{R}^{n} \times M$ and $\phi(0, \bar{m})=0$. Then, for the initial state $\left(x_{0}, m_{0}\right)=(0, \bar{m})$,

$$
\lambda=\sup _{\bar{w} \in W[0, \infty)} L(\bar{w}),
$$

and an optimal control is $w_{k}^{*}=w^{*}\left(x_{k}, m_{k}\right)$, where $w^{*}(x, m)$ achieves the maximum in (2.41).

We now relate the disturbance rejection problem to the optimal control problem (2.40) and the dynamic programming equation (2.41). First note that if $\lambda=0$, then, by Theorem 2.8, we have $\sup _{\bar{w} \in W[0, \infty)} L(\bar{w})=0$. Therefore, $L(\bar{w}) \leq 0$ for all $\bar{w} \in W[0, \infty)$ and, in particular, (2.37) is satisfied for $u$.

Theorem 2.9. Assume that there exist $a \lambda \geq 0$ and a continuous function $\phi$ so that (2.41) is satisfied for all $(x, m) \in \mathbb{R}^{n} \times M$ and $\phi(0, \bar{m})=0$. Then $\lambda=0$ if and only if $\gamma \geq \gamma^{*}$.

Proof. Assume that $\lambda=0$. Note that the function $\phi$ satisfies the inequality in (2.38). If we show that $\phi$ is nonnegative, then $\phi$ is a storage function and it follows that $\gamma \geq \gamma^{*}$. Let $K$ be given and pick $w_{k}=w^{0}$ for $k=0, \ldots, K-1$. Then it is straightforward to see that

$$
\phi(x, m) \geq \sum_{k=0}^{K-1} c^{u}\left(x_{k}, m_{k}\right)+\phi\left(x_{K}, m_{K}\right) .
$$

Let $K \geq K_{0}$, where $K_{0}$ is defined as in assumption (A). Then $m_{K}=\bar{m}$. Furthermore, since the system is undisturbed, it follows from Lemma 2.7 that for any $\tilde{\varepsilon}>0$, there exists a $\tilde{K}$ such that if $K \geq \tilde{K}$, then $\left\|x_{k}\right\| \leq \tilde{\varepsilon}$. Furthermore, it follows from the continuity of $\phi$ in $x$ that for any $\varepsilon$, there exists $\tilde{\varepsilon}$ such that $|\phi(x, m)|<\varepsilon$ whenever $\left\|x_{k}\right\| \leq \tilde{\varepsilon}$. Therefore, for $K$ large enough, we have

$$
\phi(x, m) \geq \sum_{k=0}^{K-1} c^{u}\left(x_{k}, m_{k}\right)+\phi\left(x_{K}, \bar{m}\right) \geq \phi\left(x_{K}, \bar{m}\right) \geq-\varepsilon .
$$

Since $\varepsilon$ is arbitrary, it follows that $\phi(x, m) \geq 0$ and the result follows.

Assume now that $\gamma \geq \gamma^{*}$. Then, for any $K>1, w \in W[0, K-1]$, we have, from (2.38),

$$
\begin{gathered}
\Phi(x, m) \geq \Phi\left(f^{u}(x, m), g(m, w)\right)+c^{u}(x, m)-\gamma^{2} d(w), \\
\Phi(0, \bar{m})=0 .
\end{gathered}
$$

Consider system (2.39) with initial condition $\left(x_{0}, m_{0}\right)=(0, \bar{m})$. Then it follows from (2.38) that

$$
\begin{aligned}
\sum_{k=1}^{K-1}\left(c^{u}\left(x_{k}, m_{k}\right)-\gamma^{2} d\left(w^{k}\right)\right) & \leq \sum_{k=0}^{K-1}\left(\Phi\left(x_{k}, m_{k}\right)-\Phi\left(x_{k+1}, m_{k+1}\right)\right) \\
& =\Phi\left(x_{0}, m_{0}\right)-\Phi\left(x_{K}, m_{K}\right) \\
& =-\Phi\left(x_{K}, m_{K}\right) \leq 0,
\end{aligned}
$$


and thus

$$
\limsup _{K \rightarrow \infty} \frac{1}{K} \sum_{k=1}^{K-1}\left(c^{u}\left(x_{k}, m_{k}\right)-\gamma^{2} d\left(w_{k}\right)\right) \leq 0 .
$$

Therefore, from Theorem 2.8 and (2.47), we get $\lambda \leq 0$. But, by assumption, $\lambda \geq 0$, and thus $\lambda=0$.

\section{Relationship between robust and risk-sensitive problems}

Define the optimal cost of transferring the finite-state machine from $m$ to $\hat{m}$ in one step as

$$
V(m, \hat{m})=\min _{w \in W}\{d(w): \hat{m}=g(m, w)\}
$$

For $\varepsilon>0$, define

$$
\Pi_{\varepsilon}(m, \hat{m})=\frac{1}{Z_{\varepsilon}(m)} e^{-(V(m, \hat{m}) / \varepsilon)}
$$

where $Z_{\varepsilon}(m)=\sum_{\hat{m} \in M} e^{-(V(m, \hat{m}) / \varepsilon)}$ is a normalization constant. Then $\Pi_{\varepsilon}$ is the stochastic matrix of a Markov chain on $M$. Note that since $d(w) \geq 0, d\left(w^{0}\right)=0$, and $g\left(m, w^{0}\right)=$ $h(m)$, we have

$$
\lim _{\mathcal{\varepsilon} \rightarrow 0} \Pi_{\mathcal{E}}(m, \hat{m})= \begin{cases}1 & \hat{m}=h(m), \\ 0 & \text { otherwise. }\end{cases}
$$

Therefore, the Markov chain with the stochastic matrix $\Pi_{\varepsilon}$ is a stochastic perturbation (indexed by $\varepsilon>0$ ) of the undisturbed dynamics $m_{k+1}=h\left(m_{k}\right)$.

For the stochastic system with state $\left(x_{k}, r_{k}^{\varepsilon}\right)$, where $r_{k}^{\varepsilon}$ is the Markov chain, $P\left(r_{k+1}^{\varepsilon}=\right.$ $\left.\hat{m} \mid r_{k}^{\varepsilon}=m\right)=\Pi_{\varepsilon}(m, \hat{m})$, and $x_{k}$ is given by (2.1) (with $r_{k}^{\varepsilon}$ replacing $r_{k}$ ), define the risksensitive index

$$
J_{\mathcal{\varepsilon}}(u)=\varepsilon \limsup _{K \rightarrow \infty} \frac{1}{K} \log E_{x, r}\left[e^{(1 / \varepsilon) \sum_{k=0}^{K-1} c\left(x_{k}, r_{k}^{\varepsilon}, u_{k}\right)}\right] .
$$

As in Section 2.1, we define the operator

$$
\left(P_{\varepsilon}^{c} \phi\right)(x, r)=e^{c(x, r, u) / \varepsilon}\left(P_{\varepsilon} \phi\right)(x, r)=e^{c(x, r, u) / \varepsilon} \sum_{\hat{r} \in M} \Pi(r, \hat{r}) \phi(A(r) x+B(r) u, \hat{r}) .
$$

Assume that there exist a constant $\lambda^{\varepsilon}$, a function $\psi^{\varepsilon}$, and an optimal control $u^{\varepsilon} \in \mathfrak{U}$ so that Theorem 2.1, with $\lambda^{\varepsilon} / \varepsilon$ replacing $\lambda$, holds. Then

$$
\lambda^{\varepsilon}=J^{\varepsilon}\left(u^{\varepsilon}\right)
$$


Furthermore, by (2.24), we have (with $\phi^{\varepsilon}=\varepsilon \log \psi^{\varepsilon}$ )

$$
\lambda^{\varepsilon}+\phi^{\varepsilon}(x, r)=\sup _{\pi \in \mathscr{P}(M)}\left\{\sum_{\hat{r} \in M} \phi^{\varepsilon}\left(f^{u^{\varepsilon}}(x, r), \hat{r}\right) \pi(\hat{r})+c^{\mathcal{u}^{\varepsilon}}(x, r)-\varepsilon I\left(\pi \| \Pi_{\varepsilon}(r, \cdot)\right)\right\},
$$

where $f^{u^{\varepsilon}}(x, r)=A(r) x+B(r) u^{\varepsilon}(x, r)$.

We now show that, under the appropriate conditions, in the limit $\varepsilon \rightarrow 0$, equation (3.7) for the risk-sensitive problem (3.4) converges to the corresponding equation (2.41) for the disturbance rejection problem (2.37). Recall that, by Lemma 2.2, the supremum in (3.7) is attained at the measure

$$
\pi_{x}^{*}(r, \hat{r})=\frac{e^{\phi^{\varepsilon}\left(f^{u^{\varepsilon}}(x, r), \hat{r}\right) / \varepsilon}}{\left(P_{\varepsilon} e^{\phi^{\varepsilon} / \varepsilon}\right)(x, r)} \Pi_{\varepsilon}(r, \hat{r})
$$

and

$$
\begin{aligned}
\lambda^{\varepsilon}+\phi^{\varepsilon}(x, r) & =\sup _{\pi \in \mathscr{P}(M)}\left\{\sum_{\hat{r} \in M} \phi^{\varepsilon}\left(f^{u^{\varepsilon}}(x, r), \hat{r}\right) \pi(\hat{r})+c^{u^{\varepsilon}}(x, r)-\varepsilon I\left(\pi \| \Pi_{\mathcal{\varepsilon}}(r, \cdot)\right)\right\} \\
& =\sum_{\hat{r} \in M} \phi^{\varepsilon}\left(f^{u^{\varepsilon}}(x, r), \hat{r}\right) \pi_{x}^{*}(r, \hat{r})+c^{u^{\varepsilon}}(x, r)-\varepsilon I\left(\pi_{x}^{*}(r, \cdot) \| \Pi_{\mathcal{\varepsilon}}(r, \cdot)\right) \\
& =\varepsilon \log \sum_{\hat{r} \in M} \Pi_{\mathcal{\varepsilon}}(r, \hat{r}) e^{\phi^{\varepsilon}\left(f^{u^{\varepsilon}}(x, r), \hat{r}\right) / \varepsilon}+c^{u^{\varepsilon}}(x, r) .
\end{aligned}
$$

Using a variation of the Laplace-Varadhan lemma (see, e.g., [1]), it is straightforward to see that the first term on the right-hand side of (3.9) converges in the limit $\varepsilon \rightarrow 0$ to $\max _{w \in W}\left[\phi^{0}\left(f u^{0}(x, r), g(r, w)\right)-d(w)\right]$, and therefore (3.9) becomes

$$
\lambda^{0}+\phi^{0}(x, r)=\max _{w \in W}\left[\phi^{0}\left(f^{u^{0}}(x, r), g(r, w)\right)+c^{u^{0}}(x, r)-d(w)\right] .
$$

Provided that the solution of (3.10) is unique at the control $u^{0}$, it follows that (3.10) is the same as (2.41) (evaluated at $u^{0}$ ) and, therefore, that the two problems are equivalent in the limit $\varepsilon \rightarrow 0$.

\section{Acknowledgment}

This research was supported by the US National Science Foundation under Grant no. ECS-9629866.

\section{References}

[1] J. Baras and M. James, Robust and Risk-Sensitive Output Feedback Control for Finite State Machines and Hidden Markov Models, Isr 1994-63, University of Maryland, Maryland, 1994.

[2] M. Branicky, Multiple Lyapunov functions and other analysis tools for switched and hybrid systems, IEEE Trans. Automat. Control 43 (1998), no. 4, 475-482.

[3] J. Cury, Krogh, B., and T. Niinomi, Synthesis of supervisory controllers for hybrid systems based on approximating automata, IEEE Trans. Automat. Control 43 (1998), no. 4, 564-568.

[4] P. Dupuis and R. Ellis, A Weak Convergence Approach to the Theory of Large Deviations, Wiley Series in Probability and Statistics, John Wiley \& Sons, New York, 1997. 
[5] W. Fleming and D. Hernández-Hernández, Risk-sensitive control of finite state machines on an infinite horizon. I, SIAM J. Control Optim. 35 (1997), no. 5, 1790-1810.

[6] W. Fleming and W. M. McEneaney, Risk-sensitive control on an infinite time horizon, SIAM J. Control Optim. 33 (1995), no. 6, 1881-1915.

[7] M. Ghosh, A. Arapostathis, and S. I. Marcus, Optimal control of switching diffusions with application to flexible manufacturing systems, SIAM J. Control Optim. 31 (1993), no. 5, 11831204.

[8] Ergodic control of switching diffusions, SIAM J. Control Optim. 35 (1997), no. 6, 19521988.

[9] K. Glover and J. Doyle, State-space formulae for all stabilizing controllers that satisfy an $H_{\infty}$-norm bound and relations to risk sensitivity, Systems Control Lett. 11 (1988), no. 3, 167-172.

[10] D. Hernández-Hernández and S. Marcus, Risk sensitive control of Markov processes in countable state space, Systems Control Lett. 29 (1996), no. 3, 147-155.

[11] M. Mariton, Jump Linear Systems in Automatic Control, Marcel Dekker, New York, 1990.

[12] S. Pettersson, Analysis and Design of Hybrid Systems, Ph.D. thesis, Chalmers University of Technology, Sweden, 1999.

[13] A. Rantzer and M. Johansson, Piecewise linear quadratic optimal control, IEEE Trans. Automat. Control 45 (2000), no. 4, 629-637.

[14] T. Runolfsson, Risk-sensitive control of Markov chains and differential games, Proc. 32nd Conf. on Decision and Control (Lake Buena Vista, Fla), vol. 4, 1993, pp. 3377-3378.

[15] , The equivalence between infinite-horizon optimal control of stochastic systems with exponential-of-integral performance index and stochastic differential games, IEEE Trans. Automat. Control 39 (1994), no. 8, 1551-1563.

[16] D. Šiljak, Reliable control using multiple control systems, Internat. J. Control 31 (1980), no. 2, 303-329.

[17] D. Sworder, Feedback control of a class of linear systems with jump parameters, IEEE Trans. Automat. Control 14 (1969), no. 1, 9-14.

[18] P. Whittle, Risk-Sensitive Optimal Control, Wiley-Interscience Series in Systems and Optimization, John Wiley \& Sons, Chichester, 1990.

[19] M. Wicks, P. Peleties, and R. DeCarlo, Construction of piecewise Lyapunov functions for stabilizing switched systems, Proc. 33rd Conf. on Decision and Control (Lake Buena Vista, Fla), 1994, pp. 3492-3497.

[20] A. Willsky and B. Levy, Stochastic stability research for complex power systems, Tech. Report ET-76-C-01-2295, Laboratory for Information and Decision Systems, MIT, Massachusetts, 1979.

[21] H. Ye, A. Michel, and L. Hou, Stability theory for hybrid dynamical systems, IEEE Trans. Automat. Control 43 (1998), no. 4, 461-474.

Thordur Runolfsson: School of Electrical and Computer Engineering, University of Oklahoma, Norman, OK 73019, USA

E-mail address: runolfsson@ou.edu 


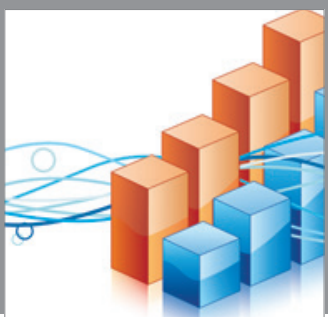

Advances in

Operations Research

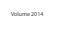

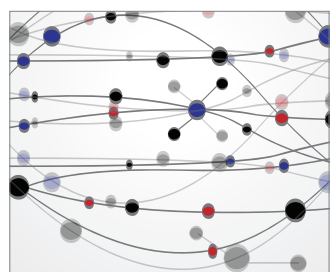

\section{The Scientific} World Journal
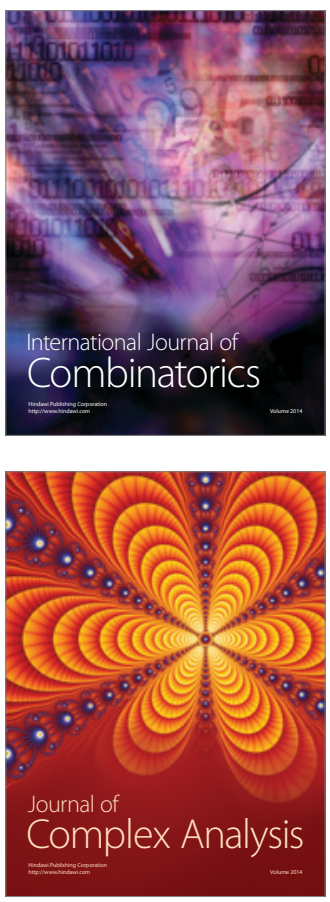

International Journal of

Mathematics and

Mathematical

Sciences
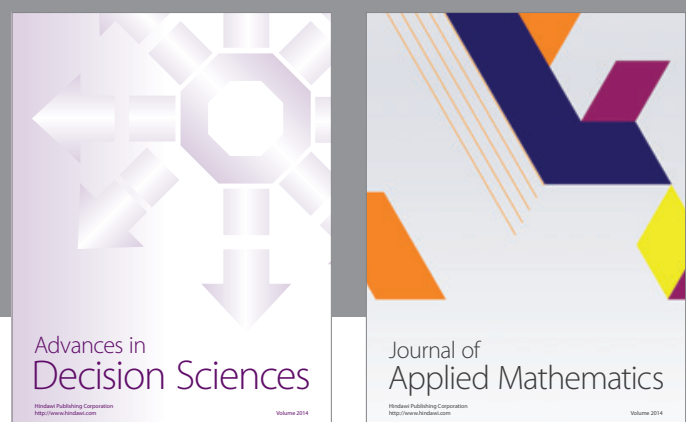

Journal of

Applied Mathematics
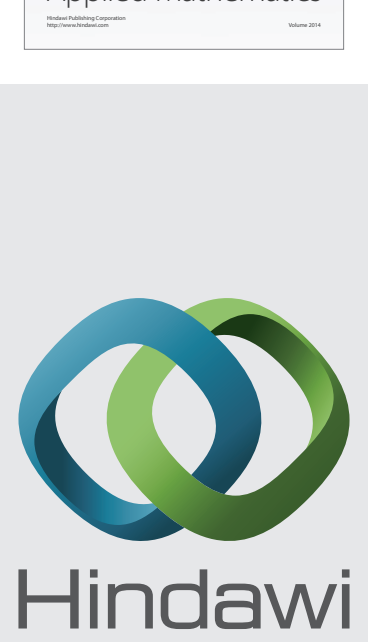

Submit your manuscripts at http://www.hindawi.com
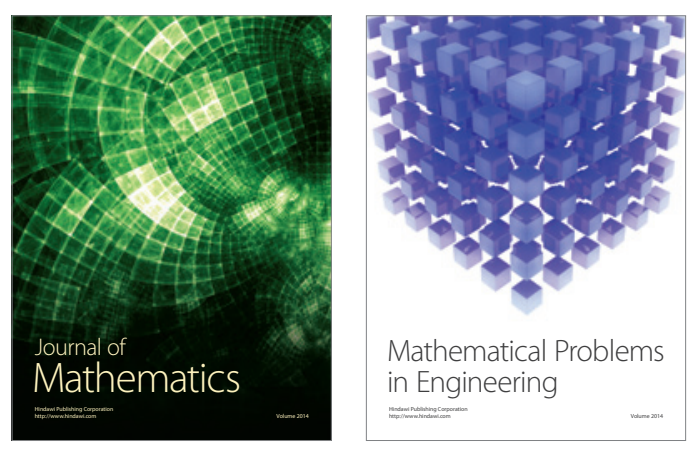

Mathematical Problems in Engineering
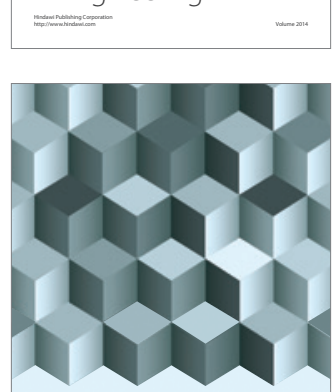

Journal of

Function Spaces
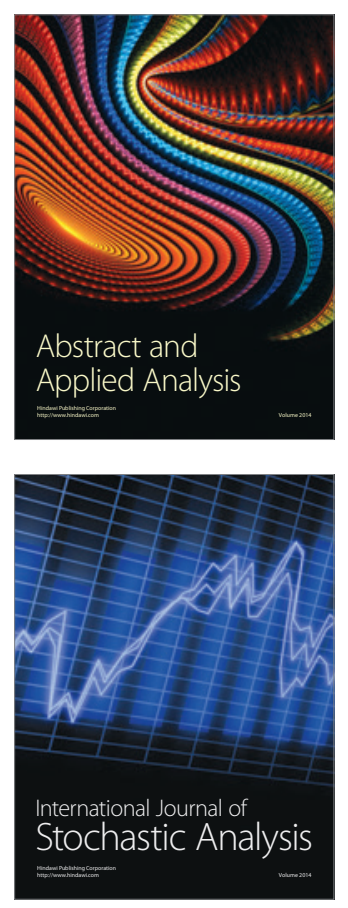

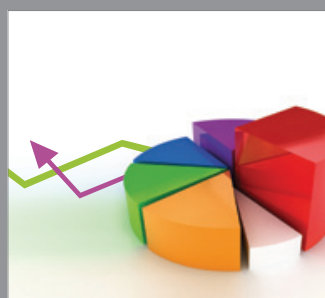

ournal of

Probability and Statistics

Promensencen
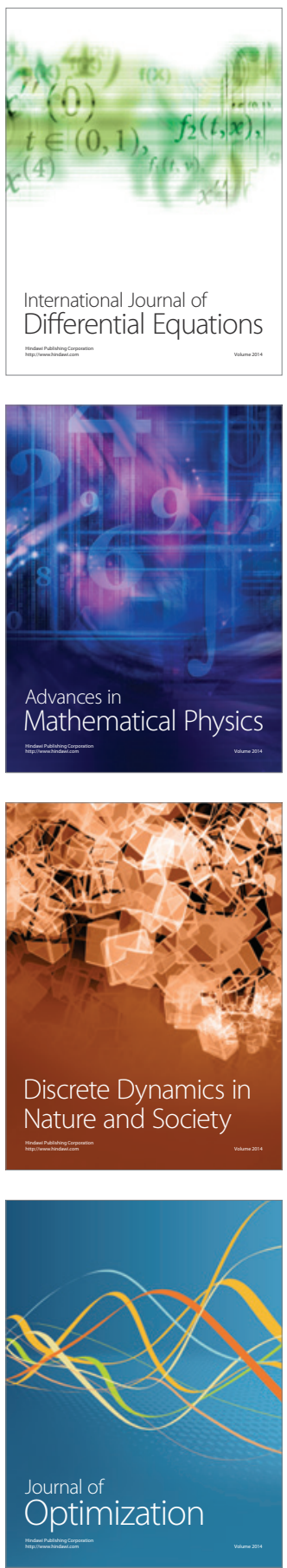